\title{
Metformin: Focus on Melanoma
}

\author{
Emilie Jaune ${ }^{1,2}$ and Stéphane Rocchi ${ }^{1,2 *}$ \\ ${ }^{1}$ INSERM U1065, Centre Méditerranéen de Médecine Moléculaire (C3M), Nice, France, ${ }^{2}$ Université de Nice Sophia Antipolis, \\ UFR de Médecine, Nice, France
}

OPEN ACCESS

Edited by:

Graham Rena,

University of Dundee, United Kingdom

Reviewed by:

Silvio Naviglio,

Università degli Studi della Campania

"Luigi Vanvitelli" Caserta, Italy

Susanna Morano,

Sapienza Università di Roma, Italy

${ }^{*}$ Correspondence: Stéphane Rocchi srocchi@unice.fr

Specialty section:

This article was submitted to Cancer Endocrinology,

a section of the journal

Frontiers in Endocrinology

Received: 09 April 2018

Accepted: 31 July 2018

Published: 21 August 2018

Citation:

Jaune E and Rocchi S (2018) Metformin: Focus on Melanoma.

Front. Endocrinol. 9:472.

doi: 10.3389/fendo.2018.00472
Metformin is the most common biguanide used in the treatment of diabetes, with 120 million treated patients worldwide. Metformin decreases hyperglycemia without inducing hypoglycemia in diabetic patients and is very well tolerated. The principal effects of metformin are to decrease hepatic gluconeogenesis and increase glucose absorption by skeletal muscles. These effects are primarily due to metformin's action on mitochondria, which requires the activation of metabolic checkpoint AMP-activated protein kinase (AMPK). AMPK is implicated in several pathways, and following metformin activation, it decreases protein synthesis and cell proliferation. Many studies have examined the role of metformin in the regulation of cancer cells, particularly its effects on cancer cell proliferation and cell death. Encouraging results have been obtained in different types of cancers, including prostate, breast, lung, and skin cancers (melanoma). Furthermore, many retrospective epidemiological studies in diabetes patients have shown that metformin treatment decreased the risk of cancers compared with other antidiabetic treatments. In this review, we will discuss the effects of metformin on melanoma cells. Together, our novel data demonstrate the importance of developing metformin and new biguanide-derived compounds as potential treatments against a number of different cancers, particularly melanoma.

\section{Keywords: biguanides, metformin, melanoma skin cancer, cancer treatment, AMPK pathway}

\section{INTRODUCTION}

Biguanides are molecules derived from guanidine and are used in diabetes treatment. Guanidine is extracted from Galega officinalis, a plant used in medicine for many years. Indeed, this plant was used for its antidiabetic properties before its effects on glycaemia were discovered in the 1920s (1). Since this time, many guanidine-derived compounds have been used in type 2 diabetes, such as buformin, phenformin, and metformin. At first, metformin was not truly compared with other guanidine-derived compounds because of its less important effects on insulin sensitivity. Other biguanides, phenformin, and buformin were widely used in diabetic treatments starting in 1920 until their high toxicity in patients was discovered in 1930 (2). Afterwards, biguanides were no longer used in type 2 diabetes treatment until a study by French chemist Jean Sterne in 1957, where he showed metformin's effects on type 2 diabetes without apparent toxicity (3). Thanks to this study, metformin received marketing authorization in Europe in 1958 and in the USA in 1995 (2). Currently, metformin is the most prescribed antidiabetic medication in the world, and it is used to treat more than 120 million people (4).

After years of treatment with metformin, retrospectives studies showed that diabetes patients had decreased cancer incidence with metformin compared to treatment with another antidiabetic drug (5). Afterwards, studies confirmed these results $(6,7)$, and many groups focused their research on metformin's effects on cancer cells. In this study, we will particularly focus on metformin's effects on melanoma. 
Cutaneous melanoma is a malignant cancer that rises from the transformation of melanocytes. These cells are normally responsible for the synthesis of melanin, which is a photoprotector pigment. Melanoma is widespread with 200,000 new cases every year and 65,000 melanoma-associated deaths. Its incidence doubles every 10 years, and although it represents only $10 \%$ of all skin cancers, melanoma is responsible for $80 \%$ of skin cancer deaths, which constitutes a real public health problem (8). Melanoma is the most aggressive skin cancer and possesses a strong invasive capability that enables the development of metastasis principally in the lymph node, liver, lung but also in the central nervous system. Metastatic melanoma is one of the deadliest cancers because of the inefficacy of current therapies.

For 15 years, targeted therapy against BRAF(mutated in 50$60 \%$ of primary melanoma) or MEK protein has been developed, and some of these treatments have been commercialized, including BRAF inhibitors, such as vemurafenib (or PLX4032) and dabrafenib, and MEK inhibitors, such as cobimetinib, or trametinib (9). The first results with these therapies seem promising with an increase in overall survival and shrinkage of the primary tumor. However, after few weeks of treatment, patients develop a strong resistance to these therapies, enabling metastatic growth and relapse $(10,11)$. Furthermore, melanoma cells have the ability to escape the immune response. Due to this observation, current therapeutic approaches try to allow immune system activation to kill melanoma cells $(12,13)$. Currently, two different antibodies are commercialized: ipilimumab (antiCTLA-4) and nivolumab (anti-PD-1). ipilimumab targets CD4+ $\mathrm{T}$ cells, whereby its inhibition allows $\mathrm{T}$-cell activation. This treatment increases patient survival rate, but only $15-20 \%$ of patients respond to this treatment (14). PD-1 is also expressed on $\mathrm{T}$ cells, and its expression inhibits T-cell activation. Its target, PDL-1, is widely found in melanoma cells. PD-1 treatment shows a response in $\sim 30-40 \%$ of patients (15). Even if these responses result in an objective and long-lasting response, $\sim 55-60 \%$ of patients do not respond to these treatments. The identification of new antimelanoma compounds is essential for developing new therapies.

\section{PRINCIPAL EFFECTS OF METFORMIN IN TYPE 2 DIABETES TREATMENTS}

In type 2 diabetes patients, metformin ( $\mathrm{N}, \mathrm{N}$-dimethylbiguanide) exerts its antidiabetic function by decreasing the insulin resistance of glucose-intolerant patients and hepatic gluconeogenesis. Indeed, the liver is considered to be the principal site of action of metformin, where it can act on gluconeogenesis, glycolysis, and glycogen synthesis. In type 2 diabetes patients, hepatic gluconeogenesis is increased relative to healthy patients. However, under metformin treatment, glucose absorption and general levels of glucose can decrease to $75 \%$ (16). Furthermore, this molecule also increases the high absorption of glucose by skeletal muscles, which improves its effects on glucose homeostasis. In general, metformin increases glucose absorption by increasing the plasma membrane translocation of glucose receptors, such as glucose transporter 1 (GLUT-1), in both hepatic cells (17) and skeletal muscle cells (18). In addition, this compound highly increases the expression of insulin receptor substrate 1 and 2 (IRS-1 and 2), which enhances glucose absorption.

Interestingly, metformin also blocks the effects of glucagon, which normally enhances gluconeogenesis, by inhibiting essential enzymes in this process and stimulating glycolysis via the alteration of numerous enzymes in this signaling pathway (19).

However, we currently do not understand all the mechanisms of actions of metformin in these patients. Interestingly, a recent study showed the effect of metformin on the intestinal microbiota and its impact on metabolism in obese mice (20). Indeed, type 2 diabetes seems to be impacted by the intestinal microbiota (21), and therefore, the effects on the microbiota could be partly responsible for metformin's effects in type 2 diabetes patients.

\section{METFORMIN ACTS AS AN ANTICANCER AGENT}

\section{Retrospective Studies}

Diabetic patients possess a higher risk of developing cancers than healthy patients, which is partly due to increasing levels of circulating growth factors, such as insulin or insulin growth factor 1 and 2 (IGF-1 and 2) (22). In this context, many retrospective analyses in type 2 diabetes patients have shown that metformin possesses antitumoral proprieties (5-7). In Evans et al. diabetic patients treated with metformin presented less cancer than patients treated with other antidiabetics. Following this study, many investigations have shown the antineoplastic effects of metformin in numerous cancer types (6, 23-25). For example, a study compared the effects of three different treatments, metformin, insulin, or sulfonylureas, over 5 years in $\sim 10,300$ diabetes patients. The results showed that patients treated with metformin have a lower cancer-related mortality rate than patients treated with other treatments (23). Inversely, the study by Currie et al. showed that patients treated with insulin developed more solid cancer than those treated with metformin (25). Another study observed that $7.3 \%$ of type 2 diabetes patients treated with metformin developed cancers compared with $11.6 \%$ of patients treated with other antidiabetics (6). In a more specific retrospective study, it was shown that the use of metformin for long-term treatment in men decreased prostate cancer development by $34 \%$ compared to patients treated with other antidiabetic drugs (26). In women, metformin treatment induced a $56 \%$ decrease in the breast cancer risk of diabetic patients (24). Recently, a study in a Korean population with type 2 diabetes showed a decrease in cancer development for patients treated with long-term metformin (5.8 years) with an incidence of 13.2 per 1000 compared with an incidence of 21.8 per 1000 in patients with another treatments (27).

In 2010, a short-term clinical study ( 1 month) performed in non-diabetic patients showed the significant effect of metformin on the development of rectal aberrant crypt foci (precancerous lesions) and the proliferation of colonic epithelial cells (28). Currently, 304 clinical trials have been registered on metformin 
treatment in different cancer types (ClinicalTrial.gov; March 2018).

\section{Mechanisms of Action of Metformin on Cancer Cells}

Consequently, many laboratories have tried to understand the mechanisms of action of metformin in different types of cancers, such as lung, prostate, and ovarian cancers or melanoma. The in vitro effects of metformin, alone or in combination with other drugs, have been studied in many different cancers (2932). Moreover, numerous in vivo studies have demonstrated the efficacy of metformin in decreasing tumoral growth $(33,34)$.

\section{Indirect Effects of Metformin}

In these studies, different mechanisms have been identified to explain metformin's effects on cancer cells. The first mechanism is an indirect effect of metformin. Indeed, in different cancers, such as breast, colon, or prostate cancer, hyperinsulinemia and obesity induced by insulin and IGF1/2 are associated with poor prognosis (35). Interestingly, metformin decreases circulating insulin levels in patients. Indeed, the transcription of key genes inhibits gluconeogenesis by metformin in the liver, and increased glucose absorption in skeletal muscle cells involves a decrease in blood glucose levels, decreasing circulating insulin levels (36). Therefore, metformin decreases tumoral growth by its inhibition of circulating insulin levels (Figure 1). Furthermore, in a mouse model, metformin inhibited lung cancer cell growth induced by hyperinsulinemia and obesity by decreasing the circulating level of insulin and by activating the AMPK pathway (37). Finally, in non-diabetic woman with breast cancer, a study showed that metformin decreased circulating insulin levels by $22 \%$ and increased insulin sensitivity by $25 \%$ (38). These results confirm that a decrease in insulin induced by metformin can be considered a new potential mechanism in metformin inhibition of tumorigenesis. As we described previously, metformin seems to impact the microbiota in type 2 diabetes patients (20). Therefore, it will be interesting to study the impact of metformin on the microbiota in different cancer types.

\section{Direct Effects of Metformin}

However, the principal effects of metformin on cancer cells are direct effects, which predominantly induce mammalian target of rapamycin complex 1 (mTORC1) inhibition (Figure 1). mTORC1 is a protein complex composed of five different proteins: DEP domain-containing mTOR interacting protein (DEPTOR), mammalian LST8/G-protein $\beta$-subunit like protein (mLST8), regulatory-associated protein of mTOR (RAPTOR), proline-rich AKT substrate of $40 \mathrm{kDa}$ (PRAS40), and mammalian target of rapamycin (mTOR). This complex is implicated in many cellular processes but principally in protein synthesis regulation, which is essential for cellular growth. This complex is often activated in cancer cells and can be associated with cancer therapy resistance. Furthermore, mTORC1 plays a critical role in the proliferation and growth of normal stem cells and cancer stem cells. mTOR's implication in cancer stem cell proliferation has been demonstrated in various cancer types, such as colon, pancreas, or breast cancer (39-41).
Depending on the cancer type, many different mechanisms have been discovered to explain the inhibition of mTORC1. The principal one induces AMPK pathway activation after mitochondria dysregulation by metformin. Indeed, at the cellular level, metformin principally acts on mitochondria by inhibiting complex I of the mitochondrial respiratory chain, which disrupts ATP production in the cell (42) and induces AMPK activation (43). A recent study also showed that metformin dysregulates mitochondrial functions via calcium flux release (44). Indeed, metformin induces endoplasmic reticulum (ER) stress, which releases calcium into the cytoplasm of the cell. This calcium release induces higher calcium absorption by the mitochondria, which results in mitochondrial swelling. AMPK is an energy sensor that plays an important role in many metabolic pathways involved in restoring energetic balance within the cell (45). In addition, when AMPK activation is sustained, it can play an important role in different cellular processes, such as cell growth and proliferation, cell cycle regulation, cell polarity, apoptosis, and autophagy (46). After metformin induction, AMPK seems to be activated in cancer cells on threonine 172 by liver kinase B1 (LKB1) (47). LKB1 is deleted in many different tumors, such as tumors in cervical or lung cancer, showing the link between LKB1 expression and cancer predisposition. The LKB1/AMPK pathway inhibits mTOR expression via the activation of tuberculous sclerosis complex 1 and 2 (TSC1 and TSC2), which induces the dysregulation of protein synthesis, thereby inhibiting tumoral cell proliferation. Interestingly, AMPK can also directly inhibit RAPTOR, a positive regulator of mTOR (48).

Furthermore, metformin can inhibit mTORC1 by AMPKindependent effects (49). Some of these effects are due to mTORC1 inhibition via recombinant activating gene (RAG) GTPase family protein inhibition (50). Indeed, RAG GTPases recruit mTORC1 via RAPTOR interactions at lysosomal surfaces, where they are activated by Ras homolog enriched in brain (RHEB). Metformin can also directly inhibit Ragulator (51). In prostate cancer, it has been shown that metformin can induce cancer cell death by p53/regulation in development and DNA damage responses 1 (REDD1) pathway activation, which induces the inhibition of mTOR, thereby inhibiting tumor growth (52).

Metformin inhibits cancer cell proliferation by mTORC1independent mechanisms. Indeed, AMPK can directly phosphorylate p53 on serine 15 , which increases p21 expression and enhances cell cycle arrest (53). It has also been described that metformin-induced cell cycle arrest is mediated by cyclin $\mathrm{D} 1$ inhibition and $\mathrm{Rb}$ dephosphorylation in prostate cancer cells (29) or by an AMPK-dependent mechanism requiring the downregulation of cyclin D1 and implication of p21 and p27 in breast cancer cells (54). A new mechanism implicating the upregulation of micro-RNA34a in renal cancer cells has been described to induce G0-G1 cell cycle arrest under metformin treatment (55). Furthermore, metformin-induced G1-cell cycle arrest has also been observed in pancreatic, glioma, endometrial, and ovarian cancer cells (56). Recent studies in glioblastoma and ovarian cancer cells have also shown cell cycle arrest in G2/M induced by metformin $(57,58)$. In addition, metformin can induce cell cycle arrest in the $S$ phase in triple-negative breast cancer (54). Metformin inhibits different genes implicated in 


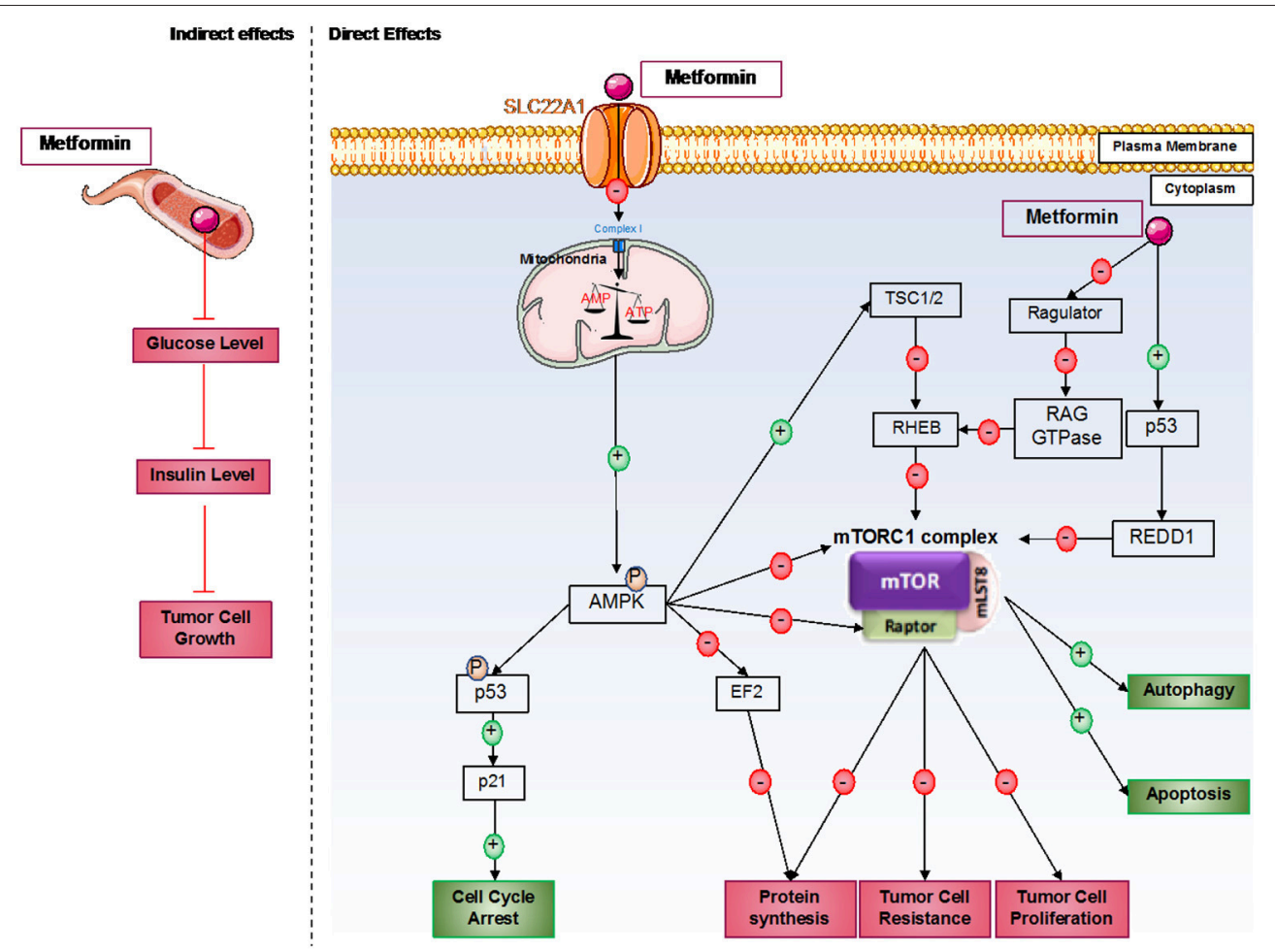

FIGURE 1 | Direct and indirect mechanisms of action of metformin in cancer cells. Metformin induces antitumorigenic effects by both indirect and direct mechanisms. In the blood circulation, metformin decreases glucose levels and therefore insulin levels; insulin can act as a growth factor in tumor cells. For direct effects, metformin acts by AMPK-dependent and independent effects. Generally, metformin induces inhibition of the mTORC1 pathway, which involves an essential protein complex in cellular processes, including protein synthesis and cell proliferation; this complex also promotes tumor cell resistance to therapies. Furthermore, metformin induces cell cycle arrest by p53 activation.

cell division, such as genes encoding tubulin or histones, which enhances cell cycle arrest (56). AMPK also inhibits protein synthesis via the inhibition of elongation factor 2 (EF2) protein (59). Furthermore, under AICAR stimulation, active AMPK can decrease fatty acid synthase (FAS) expression in prostate cancer cells (60). This enzyme is essential for fatty acid synthesis, which is also essential for cell proliferation.

Finally, the inhibition of mTORC1 also induces cell death mechanism activation, which induces cancer cell death. For the autophagy process, mTORC1 inhibits the initial step of phagophore formation. This complex also inhibits unc-51 like autophagy activating kinase 1 (ULK1), an essential kinase for autophagy induction (61). Activated AMPK induced by metformin enhances autophagy initiation via inhibition of the mTORC1 complex by phosphorylation of TSC2 on serine 1345 $(62,63)$. AMPK directly phosphorylates ULK1 and induces mTOR-independent autophagy (61). For the apoptosis process in cancer cells, it has been shown that autophagy induction enhances caspase-dependent apoptosis (64). In adipocytes and under AICAR stimulation, AMPK activates apoptosis processes via eukaryotic initiation factor $2 \alpha$ (eIF2 $\alpha$ ) regulation (65). Moreover, activation of AMPK stimulates the phosphorylation of p53 on serine 46, which is essential in apoptotic type I programmed cell death induction (66). Different studies in the pancreas and in endometrial cancers showed that the antitumor effects of metformin involve the induction of AMPK-dependent apoptosis (67). Finally, the LKB1/AMPK pathway activated by nutrient deprivation increases cyclin-dependent kinase inhibitor $1 \mathrm{~B}$ (p27), which enhances autophagy and apoptosis processes in cancer cells (68).

In addition, AMPK activation by metformin induces many different antitumor effects via the inhibition of c-MYC or hypoxia-inducible factor- $1 \alpha(\mathrm{HIF}-1 \alpha)$ (69). Metformin activates the DNA damage reparation pathway via ataxia telangiectasia mutated (ATM) activation, which inhibits tumor growth (70).

In each case, metformin acts as a major metabolism disruptor in cancer cells, induces dysregulation in energetic metabolism and protein synthesis, and activates autophagy and apoptosis processes.

\section{METFORMIN AND MELANOMA}

Metformin can induce cancer cell death by different mechanisms. However, what is known about metformin in melanoma cells?

As previously described, melanoma is the most aggressive form of skin cancer, and currently, efficient treatments have not been developed for most patients. The discovery of new treatments for this cancer appears to be essential. In this context, different laboratories, including ours, have shown that 


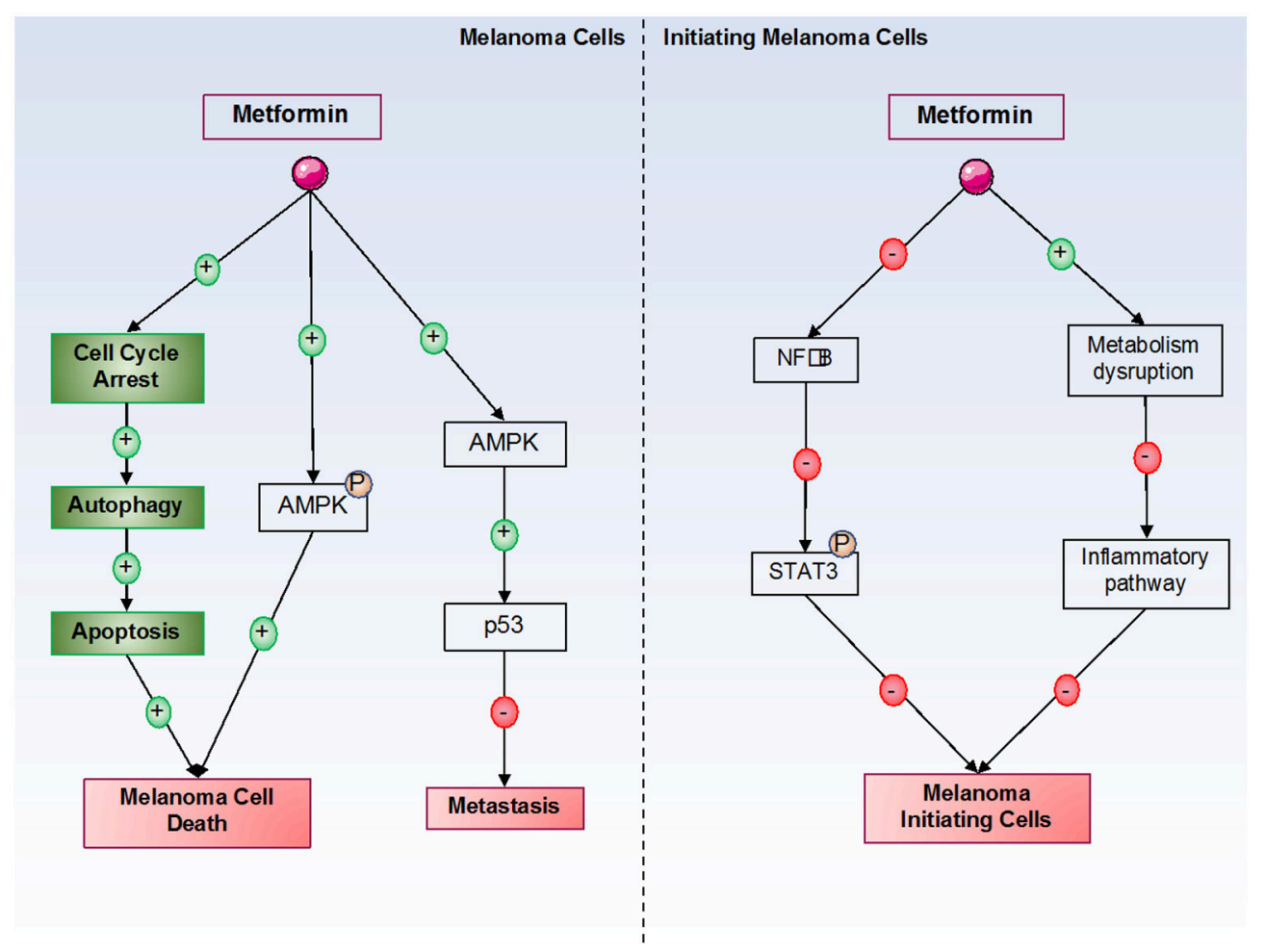

FIGURE 2 | Metformin's effects on melanoma cells. Metformin induces melanoma cell death by both AMPK-dependent and -independent pathways. By an unknown mechanism, metformin induces cell cycle arrest in melanoma cells, which is responsible for the activation of autophagy, and in turn, for the activation of apoptosis, leading to melanoma cell death. In initiating melanoma cells, metformin decreased cell transformation and proliferation by inhibiting the NF- $\mathrm{B}$ pathway and the inflammatory pathway.

metformin or phenformin (another biguanide compound) can inhibit melanoma cell proliferation $(33,71-73)$.

As previously discussed, metformin can inhibit cancer cell proliferation and induce cancer cell death by many different mechanisms. In melanoma cells, it has been shown that metformin induces cell cycle arrest in melanoma cells in the G0-G1 phase after $24 \mathrm{~h}$ of treatment at $10 \mathrm{mM}$. However, the molecular mechanism of this cell cycle arrest has not been identified in melanoma cells. Furthermore, our laboratory has also shown that this cell cycle arrest is responsible for autophagy (at $72 \mathrm{~h}$ ) and apoptosis (at $96 \mathrm{~h}$ ) induction by metformin in melanoma cells $(71,74)$. In this model, we also showed that inhibition of AMPK (by siRNA) induces a partial restoration of melanoma cell viability under metformin treatment, suggesting that AMPK plays a partial role in metformin-induced melanoma cell death. This finding also suggests that another AMPKindependent mechanism is implicated in metformin-induced melanoma cell death (Figure 2). Interestingly, in xenograft mouse models, metformin decreases the tumoral volume of melanoma cells. In addition, no cellular death has been observed in normal human cells, such as melanocytes, even if endogenous AMPK is expressed. Similar results have also been observed by other laboratories $(75,76)$. In another study, metformin induced autophagy activation in melanoma cells by inhibiting a new potential therapeutic target, tribbles pseudokinase 3 (TRIB3)
(33). In this study, the authors showed that metformin attenuated melanoma growth and metastasis by reducing TRIB3 expression in non-diabetic and diabetic mouse models.

Interestingly, a recent study showed that metformin can directly act not only on melanoma cells to induce cell death but also on the tumor microenvironment, particularly in the context of an immune response (77). This study showed that metformin activated both autophagy and apoptosis in melanoma cancer cells in vitro and confirmed the results in vivo in mouse models challenged with B16 murine melanoma cells. The results showed that metformin activity on melanoma cells was partly due to the immune system and that the antitumor activity of metformin was lost on immunodeficient (NSG) mice. This group also showed that metformin interaction with the immune system was principally associated with T cells (77). As described in the introduction, the immune system is very important in melanoma therapies, and current immunotherapies show very interesting objective responses, but they occur in very few patients. This study showed the interactions between the immune system and metformin; thus, it will be interesting to test a combination of metformin treatment and immunotherapies, such as anti-PD1, to increase the effects of immunotherapies in melanoma cells.

Another study from our laboratory showed that metformin inhibited the proliferation of melanoma cells (78). Indeed, in this study, we showed in vitro that metformin modulates 
the expression of different proteins, such as Slug, Snail, and matrix metalloproteinases 2 and 9 (MMP2 and 9), the latter two of which are implicated in the epithelial-mesenchymal transition via an AMPK- and p53-dependent mechanism. However, even if metformin can inhibit cell invasion, it has no effect on the migration ability of melanoma cells. Furthermore, in vivo, we showed that metformin inhibits melanoma metastasis development in the lung. In general, a study on UV-induced skin cancers showed that metformin, by an AMPK-dependent pathway, inhibited tumorigenesis in skin cells (79). Indeed, the authors showed that AMPK was inhibited by UVB irradiation, demonstrating its important role as a tumor suppressor in skin cancers. This inhibition enhanced decrease of the DNA damage response pathway via reduction of xeroderma pigmentosum C (XPC). However, under stimulation with AICAR and metformin, the DNA damage response pathway was reactivated, decreasing in cancer cell development. Therefore, the authors showed the importance of AMPK activation by different treatments, such as metformin, in decreasing cancer cell development and proliferation induced by UVB, such as in melanoma development.

Interestingly, melanoma is one of cancer that is the most dependent on and impacted by metabolism (80). Melanoma is a cancer that requires glycolytic metabolism, which is mediated by mitochondrial activity (81). Moreover, resistance to BRAF inhibitor cells have shown increased oxidative metabolism and mitochondrial dependence for cell survival (82). Therefore, in both sensitive and resistant to BRAF inhibitor melanoma cells, mitochondria, and metabolism appear to be essential, and a drug, such as metformin or another biguanide, that alters this metabolism could be an interesting prospect for new melanoma treatments. This information suggests that testing a drug such as metformin, which disrupts metabolism, in combination with other therapies, such as targeted therapies (BRAF inhibitors) or immunotherapies (anti-PD1) in melanoma cells, could increase objective responses and inhibit primary or acquired resistance to these treatments.

Studies have examined the effects of metformin in combination with BRAF inhibitors, such as vemurafenib (Zelboraf). Indeed, many groups have used combination treatments with BRAF inhibitors to inhibit resistance in melanoma cells. The combination of vemurafenib (BRAF inhibitor) and metformin showed encouraging results with synergistic effects for inducing melanoma cell death (76). Indeed, in vitro experiments show synergistic antiproliferative effects, particularly in BRAFV600E mutant cell lines. In other studies, metformin increased the toxicity of cisplatin, a chemotherapeutic drug, in melanoma cells $(83,84)$.

These results seem interesting, but further study is needed. Indeed, in certain studies, the combination of metformin and a BRAF inhibitor stimulated the proliferation of melanoma cells mutated by NRAS (76). It will be interesting to observe the metabolic characteristics of melanoma cells after a treatment combination of metformin and BRAF inhibitors to better understand active mechanisms.
Furthermore, metformin effects were analyzed in combination with immunotherapies (anti-CTLA4, anti-PD-1 or anti-PD-L1). As previously stated, immunotherapies have been developed for melanoma treatment for a few years. These therapies, which tend to reactivate the patient's immune system, show very efficient and durable responses, but they are effective in only $15-30 \%$ of patients. Therefore, we can imagine that combinations with another molecule, such as metformin or another biguanide can increase the objective responses obtained with immunotherapies and decrease resistance to these treatments. Interestingly, a recent study showed that phenformin, another biguanide, potentiated the effect of immunotherapy (85). In this study, the authors showed that phenformin induced the production of reactive oxygen species in granulocytic myeloid-derived suppressor cells, which increased the effect of immunotherapies on melanoma cells. Indeed, in combination with anti-PD-1, phenformin enhanced melanoma inhibition in a BRAF V600E/PTEN-null melanoma mouse model. In these mice, CD8+ lymphocytes were activated, which increased melanoma cell death. In addition, results from the study by Scharping et al. suggested that tumor hypoxia plays a role as a barrier against immunotherapy and that metformin, which can reduce intratumoral hypoxia, can improve immunotherapy efficacy against melanoma cells (86). Taken together, these results suggest that biguanides, such as metformin, could be used in combination with targeted therapies against BRAF or with immunotherapies to synergize treatment effects on melanoma cells.

Finally, certain studies have examined metformin's effects on melanoma initiating cells (MIC). Indeed, melanoma is a heterogenic tumor, and some studies including ours have suggested that MIC could be responsible for the metastatic potential of melanoma, which could be implicated in resistance to BRAF inhibitor therapies $(87,88)$. These MIC constitute a chemoresistant cell population that expresses specific markers. However, independently of MIC numbers, characteristics, or mechanisms that regulate the transition between MIC and proliferative cells, it is clear that melanoma cell populations with different tumorigenic potentials exist (89). A study has shown that STAT3 (Signal Transducer and Activator of Transcription 3) pathway activation is necessary to acquire "MIC properties" (90). Furthermore, a recent study showed that a combination of stattic and metformin decreased brain tumor initiating cells by STAT3dependent mechanisms (91). Interestingly, metformin blocks the inflammatory pathway responsible for stem cell transformation and growth due to cellular metabolism disruption (84). Finally, our laboratory has shown that metformin can decrease MIC populations (unpublished results) (Figure 2).

\section{CLINICAL TRIALS OF METFORMIN ON MELANOMA TREATMENT}

In this context, 304 clinical trials have been or are currently being performed to test the effects of metformin on different cancer types (ClinicalTrials.gov).

In our laboratory, thanks to the in vitro and in vivo results obtained from metformin treatment against melanoma, 
we developed a phase II clinical trial that was performed in the dermatology department at the University Hospital Centre in Nice. This clinical trial allowed us to determine the efficacy of metformin treatment on metastatic melanoma patients (92). In this study, patients were experiencing therapeutic failure to chemotherapies and BRAF inhibitors and were not eligible for or not responsive to immunotherapy treatment. The study was performed on 17 patients with ages ranging from 49 to 88 years (mean of 74 years). Metformin was prescribed at $1,000 \mathrm{mg}$ three times daily. After 4 months, 11 patients showed melanoma progression, 3 patients were deceased due to the disease and 2 patients had to stop treatment. After 6 months, the only patient still being treated with metformin did not show a significant response. These results were not very encouraging; it could have been concluded that metformin treatment at similar doses to those in type 2 diabetes did not induce significant efficacy in this population of metastatic melanoma patients, independently of mutational status. However, the poor efficacy of metformin treatment could be linked to different barriers in this study. Indeed, treated patients were in total therapeutic failure and had notable progression of melanoma disease. Therefore, it will be interesting to test metformin or new biguanide-derived compounds with better efficacy at an early stage of disease progression.

Currently, other clinical trials are still in progress, and their results will be truly important for understanding metformin treatments against melanoma and continuing their use. Another phase I/II clinical trial is currently being performed in the United States in Louisville, and metformin's treatment effects

\section{REFERENCES}

1. Watanabe C. Studies in the metabolic changes induced by administration of guanidine bases. Influence of injected guanidine hydrochloride upon blood sugar content. J Biol Chem. (1918).

2. Pasik C, editor. Diabetes and the biguanides: the mystery of each. In: Glucophage: Serving Diabetology for 40 Years. Lyon: Groupe Lipha (1997). p. 79.

3. Sterne J. Du nouveau dans les antidiabétiques. La NN dimethylamine guanyl guanidine (NNDG). Maroc Med. (1957).

4. Bridgeman SC, Ellison GC, Melton PE, Newsholme P, Mamotte C. Epigenetic effects of metformin: from molecular mechanisms to clinical implications. Diabetes Obes Metab. (2018) 20:1553-62. doi: 10.1111/dom. 13262

5. Evans JM, Donnelly LA, Emslie-Smith AM, Alessi DR, Morris AD. Metformin and reduced risk of cancer in diabetic patients. BMJ (2005) 330:1304-5. doi: 10.1136/bmj.38415.708634.F7

6. Libby G, Donnelly LA, Donnan PT, Alessi DR, Morris AD, Evans JM. New users of metformin are at low risk of incident cancer: a cohort study among people with type 2 diabetes. Diabetes Care (2009) 32:1620-5. doi: $10.2337 / \mathrm{dc} 08-2175$

7. Monami M, Marchionni N, Mannucci E. Glucagon-like peptide-1 receptor agonists in type 2 diabetes: a meta-analysis of randomized clinical trials. Eur $J$ Endocrinol. (2009) 160:909-17. doi: 10.1530/EJE-09-0101

8. Demierre M-F. Epidemiology and prevention of cutaneous melanoma. Curr Treat Options Oncol. (2006) 7:181-6. doi: 10.1007/s11864-006-0011-z

9. Smalley, K. S. (2010). PLX-4032, a small-molecule B-Raf inhibitor for the potential treatment of malignant melanoma. Curr Opin Invest Drugs (2000) 11:699-706. in combination with vemurafenib, a BRAF inhibitor, is being evaluated in 55 patients with BRAF-mutated melanoma (ClinicalTrials.gov, NCT01638676). At the University of Louisville, another clinical trial evaluating the combination of dabrafenib/or trametinib and metformin is being evaluated. This phase I/II clinical trial started in 2014 on 53 participants, and no results have been published (ClinicalTrials.gov, NCT02143050). Other clinical trials are currently in progress to evaluate the effects of metformin in combination with different treatments; a study of metformin combined with pembrolizumab (immunotherapy) is being conducted in Pittsburgh (ClinicalTrials.gov, NCT03311308), and a study on metformin combined with dacarbazine (chemotherapy) is being conducted in Petersburg (ClinicalTrials.gov, NCT02190838).

In conclusion, metformin, and more generally biguanides, seem to be good candidates for the development of new therapies against melanoma. Their impact on metabolism and the activation of cell death mechanisms in melanoma cells could be promising in melanoma treatment. Furthermore, studies in which metformin, or other biguanides, is combined with current therapies show a synergistic response in melanoma cells, and therefore, their results could be interesting for the development of new therapy combinations against melanoma and even other cancer cell types.

\section{AUTHOR CONTRIBUTIONS}

EJ wrote the first draft and made the figures of the review. SR oversaw the work and made the corrections and the final modifications.

10. Aplin AE, Kaplan FM, Shao Y. Mechanisms of resistance to RAF inhibitors in melanoma. J Invest Dermatol. (2011) 131:1817-20. doi: 10.1038/jid.2011.147

11. Flaherty KT, Infante JR, Daud A, Gonzalez R, Kefford RF, Sosman J, et al. Combined BRAF and MEK inhibition in melanoma with BRAF V600 mutations. N Engl J Med. (2012) 367:1694-703. doi: 10.1056/NEJMoa1210093

12. Zeiser R, Schnitzler M, Andrlova H, Hellige T, Meiss F. Immunotherapy for malignant melanoma. Curr Stem Cell Res Ther. (2012) 7:217-28. doi: $10.2174 / 157488812799859883$

13. Eggermont AM, Spatz A, Robert C. Cutaneous melanoma. Lancet (2014) 383:816-27. doi: 10.1016/S0140-6736(13)60802-8

14. Weber JS, O'day S, Urba W, Powderly J, Nichol G, Yellin M, et al. Phase I/II study of ipilimumab for patients with metastatic melanoma. J Clin Oncol. (2008) 26:5950-6. doi: 10.1200/JCO.2008.16.1927

15. Brahmer JR, Drake CG, Wollner I, Powderly JD, Picus J, Sharfman WH, et al. Phase I study of single-agent anti-programmed death1 (MDX-1106) in refractory solid tumors: safety, clinical activity, pharmacodynamics, and immunologic correlates. J Clin Oncol. (2010) 28:3167-75. doi: 10.1200/JCO.2009.26.7609

16. Stumvoll M, Nurjhan N, Perriello G, Dailey G, Gerich JE. Metabolic effects of metformin in non-insulin-dependent diabetes mellitus. N Engl J Med. (1995) 333:550-4. doi: 10.1056/NEJM199508313330903

17. Gunton JE, Delhanty PJ, Takahashi SI, Baxter RC. Metformin rapidly increases insulin receptor activation in human liver and signals preferentially through insulin-receptor substrate-2. J Clin Endocrinol Metab. (2003) 88:1323-32. doi: 10.1210/jc.2002-021394

18. Fischer Y, Thomas J, Rösen P, Kammermeier H. Action of metformin on glucose transport and glucose transporter GLUT1 and GLUT4 in heart muscle cells from healthy and diabetic rats. Endocrinology (1995) 136:412-20. doi: 10.1210/endo.136.2.7835271 
19. Fulgencio JP, Kohl C, Girard J, Pégorier JP. Effect of metformin on fatty acid and glucose metabolism in freshly isolated hepatocytes and on specific gene expression in cultured hepatocytes. Biochem Pharmacol. (2001) 62:439-46. doi: 10.1016/S0006-2952(01)00679-7

20. Lee H, Lee Y, Kim J, An J, Lee S, Kong H, et al. Modulation of the gut microbiota by metformin improves metabolic profiles in aged obese mice. Gut Microbes (2017) 9:155-65. doi: 10.1080/19490976.2017.1405209

21. Forslund K, Hildebrand F, Nielsen T, Falony G, Le Chatelier E, Sunagawa $\mathrm{S}$, et al. Disentangling type 2 diabetes and metformin treatment signatures in the human gut microbiota. Nature (2015) 528:262-6. doi: 10.1038/nature 15766

22. Giovannucci E, Harlan DM, Archer MC, Bergenstal RM, Gapstur SM, Habel LA, et al. Diabetes and cancer: a consensus report. CA Cancer J Clin. (2010) 60:207-21. doi: 10.3322/caac.20078

23. Bowker SL, Majumdar SR, Veugelers P, Johnson JA. Increased cancer-related mortality for patients with type 2 diabetes who use sulfonylureas or insulin. Diabetes Care (2006) 29:254-8. doi: 10.2337/diacare.29.02.06.dc05-1558

24. Bodmer M, Meier C, Krähenbühl S, Jick SS, Meier CR. Long-term metformin use is associated with decreased risk of breast cancer. Diabetes Care (2010) 33:1304-8. doi: 10.2337/dc09-1791

25. Currie CJ, Poole CD, Jenkins-Jones S, Gale EA, Johnson JA, Morgan CL. Mortality after incident cancer in people with and without type 2 diabetes: impact of metformin on survival. Diabetes Care (2012) 35, 299-304. doi: $10.2337 / \mathrm{dc} 11-1313$

26. Murtola TJ, Tammela TLJ, Lahtela J, Auvinen A. Antidiabetic medication and prostate cancer risk: a population-based case-control study. Am J Epidemiol. (2008) 168:925-31. doi: 10.1093/aje/kwn190

27. Kim HJ, Lee S, Chun KH, Jeon JY, Han SJ, Kim DJ, et al. Metformin reduces the risk of cancer in patients with type 2 diabetes: an analysis based on the Korean National Diabetes Program Cohort. Medicine (2018) 97:e0036. doi: 10.1097/MD.0000000000010036

28. Hosono K, Endo H, Takahashi H, Sugiyama M, Sakai E, Uchiyama $\mathrm{T}$, et al. Metformin suppresses colorectal aberrant crypt foci in a short-term clinical trial. Cancer Prev Res. (2010) 3:1077-83. doi: 10.1158/1940-6207.CAPR-10-0186

29. Ben Sahra I, Laurent K, Loubat A, Giorgetti-Peraldi S, Colosetti P, Auberger $\mathrm{P}$, et al. The antidiabetic drug metformin exerts an antitumoral effect in vitro and in vivo through a decrease of cyclin D1 level. Oncogene (2008) 27:3576-86. doi: $10.1038 /$ sj.onc. 1211024

30. Aljada A, Mousa SA. Metformin and neoplasia: implications and indications. Pharmacol Ther. (2012) 133:108-15. doi: 10.1016/j.pharmthera.2011.09.004

31. Valtorta S, Dico AL, Raccagni I, Gaglio D, Belloli S, Politi LS, et al. Metformin and temozolomide, a synergic option to overcome resistance in glioblastoma multiforme models. Oncotarget (2017) 8:113090-104. doi: 10.18632 /oncotarget.23028

32. Roos JF, Qudsi M, Samara A, Rahim MM, Al-Bayedh SA, and Ahmed H. Metformin for lung cancer prevention and improved survival: a novel approach. Eur. J. Cancer Prev. (2018) doi: 10.1097/CEJ.0000000000000442

33. Li K, Zhang TT, Wang F, Cui B, Zhao CX, Yu JJ, et al. Metformin suppresses melanoma progression by inhibiting KAT5-mediated SMAD3 acetylation, transcriptional activity and TRIB3 expression. Oncogene (2018) 37:2967-81. doi: 10.1038/s41388-018-0172-9

34. Zhang JW, Zhao F, Sun Q. Metformin synergizes with rapamycin to inhibit the growth of pancreatic cancer in vitro and in vivo. Oncol Lett. (2018) 15:1811-6. doi: 10.3892/ol.2017.7444

35. Pollak M. The insulin and insulin-like growth factor receptor family in neoplasia: an update. Nat Rev Cancer (2012) 12:159-69. doi: 10.1038/ $\operatorname{nrc} 3215$

36. Cusi K, Consoli A, Defronzo RA. Metabolic effects of metformin on glucose and lactate metabolism in noninsulin-dependent diabetes mellitus. J Clin Endocrinol Metab. (1996) 81:4059-67.

37. Algire C, Zakikhani M, Blouin M-J, Shuai JH, Pollak M. Metformin attenuates the stimulatory effect of a high-energy diet on in vivo LLC1 carcinoma growth. Endocr Relat Cancer (2008) 15:833-9. doi: 10.1677/ERC08-0038

38. Goodwin PJ, Pritchard KI, Ennis M, Clemons M, Graham M, Fantus IG. Insulin-lowering effects of metformin in women with early breast cancer. Clin Breast Cancer (2008) 8:501-5. doi: 10.3816/CBC.2008.n.060
39. Cai Z, Ke J, He X, Yuan R, Chen Y, Wu X, et al. Significance of mTOR signaling and its inhibitor against cancer stem-like cells in colorectal cancer. Ann Surg Oncol. (2014) 21:179-88. doi: 10.1245/s10434-013-3146-8

40. Zeng J-Y, Sharma S, Zhou Y-Q, Yao H-P, Hu X, Zhang R, et al. Synergistic activities of MET/RON inhibitor BMS-777607 and mTOR inhibitor AZD8055 to polyploid cells derived from pancreatic cancer and cancer stem cells. Mol Cancer Ther. (2014) 13:37-48. doi: 10.1158/1535-7163.MCT-13-0242

41. Karthik G-M, Ma R, Lövrot J, Kis LL, Lindh C, Blomquist L, et al. mTOR inhibitors counteract tamoxifen-induced activation of breast cancer stem cells. Cancer Lett. (2015) 367:76-87. doi: 10.1016/j.canlet.2015.07.017

42. Miller RA, Birnbaum MJ. An energetic tale of AMPK-independent effects of metformin. J Clin Invest. (2010) 120:2267-70. doi: 10.1172/JCI43661

43. Zhou G, Myers R, Li Y, Chen Y, Shen X, Fenyk-Melody J, et al. Role of AMPactivated protein kinase in mechanism of metformin action. J Clin Invest. (2001) 108:1167-74. doi: 10.1172/JCI13505

44. Loubiere C, Clavel S, Gilleron J, Harisseh R, Fauconnier J, Ben-Sahra I, et al. The energy disruptor metformin targets mitochondrial integrity via modification of calcium flux in cancer cells. Sci Rep. (2017) 7:5040. doi: 10.1038/s41598-017-05052-2

45. Shaw RJ, Kosmatka M, Bardeesy N, Hurley RL, Witters LA, Depinho RA, et al. The tumor suppressor LKB1 kinase directly activates AMP-activated kinase and regulates apoptosis in response to energy stress. Proc Natl Acad Sci USA. (2004) 101:3329-35. doi: 10.1073/pnas.0308061100

46. Luo Z, Zang M, Guo W. AMPK as a metabolic tumor suppressor: control of metabolism and cell growth. Future Oncol. (2010) 6:457-70. doi: $10.2217 /$ fon. 09.174

47. Hawley SA, Boudeau J. Reid JL, Mustard KJ, Udd L, Mäkelä TP, et al. (2003). Complexes between the LKB1 tumor suppressor, STRAD alpha/beta and $\mathrm{MO} 25$ alpha/beta are upstream kinases in the AMP-activated protein kinase cascade. J. Biol. 2:28. doi: 10.1186/1475-4924-2-28

48. Gwinn DM, Shackelford DB, Egan DF, Mihaylova MM, Mery A, Vasquez DS, et al. AMPK phosphorylation of raptor mediates a metabolic checkpoint. Mol Cell (2008) 30:214-26. doi: 10.1016/j.molcel.2008.03.003

49. Ikhlas S, Ahmad M. Metformin: Insights into its anticancer potential with special reference to AMPK dependent and independent pathways. Life Sci. (2017) 185:53-62. doi: 10.1016/j.lfs.2017.07.029

50. Kalender A, Selvaraj A, Kim SY, Gulati P, Brûlé S, Viollet B, et al. (2010). Metformin, independent of AMPK, inhibits mTORC1 in a rag GTPasedependent manner. Cell Metab. 11, 390-401. doi: 10.1016/j.cmet.2010.03.014

51. Pierotti MA, Berrino F, Gariboldi M, Melani C, Mogavero A, Negri T, et al. Targeting metabolism for cancer treatment and prevention: metformin, an old drug with multi-faceted effects. Oncogene (2013) 32:1475-87. doi: 10.1038/onc. 2012.181

52. Ben Sahra I, Regazzetti C, Robert G, Laurent K, Le Marchand-Brustel $\mathrm{Y}$, Auberger P, et al. Metformin, independent of AMPK, induces mTOR inhibition and cell-cycle arrest through REDD1. Cancer Res. (2011) 71:436672. doi: 10.1158/0008-5472.CAN-10-1769

53. Jones RG, Plas DR, Kubek S, Buzzai M, Mu J, Xu Y, et al. AMP-activated protein kinase induces a p53-dependent metabolic checkpoint. Mol Cell (2005) 18:283-93. doi: 10.1016/j.molcel.2005.03.027

54. Alimova IN, Liu B, Fan Z, Edgerton SM, Dillon T, Lind SE, et al. Metformin inhibits breast cancer cell growth, colony formation and induces cell cycle arrest in vitro. Cell Cycle (2009) 8:909-15. doi: 10.4161/cc.8.6.7933

55. Xie W, Wang L, Sheng H, Qiu J, Zhang D, Zhang L, et al. Metformin induces growth inhibition and cell cycle arrest by upregulating microRNA34a in renal cancer cells. Med Sci Monit. (2017) 23:29-37. doi: 10.12659/MSM. 898710

56. Oliveras-Ferraros C, Vazquez-Martin A, Menendez JA. Genome-wide inhibitory impact of the AMPK activator metformin on [kinesins, tubulins, histones, auroras and polo-like kinases] M-phase cell cycle genes in human breast cancer cells. Cell Cycle (2009) 8:1633-6. doi: 10.4161/cc.8.10.8406

57. Adeberg S, Bernhardt D, Harrabi SB, Nicolay NH, Hörner-Rieber J, König L, et al. Metformin enhancedin vitroradiosensitivity associates with g2/m cell cycle arrest and elevated adenosine-5'-monophosphate-activated protein kinase levels in Glioblastoma. Radiol Oncol. (2017) 51:431-7. doi: 10.1515/raon-2017-0042

58. Fu YL, Zhang QH, Wang XW, He H. Antidiabetic drug metformin mitigates ovarian cancer SKOV3 cell growth by triggering G2/M cell cycle arrest and 
inhibition of m-TOR/PI3K/Akt signaling pathway. Eur Rev Med Pharmacol Sci. (2017) 21:1169-75.

59. Horman S, Browne G, Krause U, Patel J, Vertommen D, Bertrand L, et al. Activation of AMP-activated protein kinase leads to the phosphorylation of elongation factor 2 and an inhibition of protein synthesis. Curr Biol. (2002) 12:1419-23. doi: 10.1016/S0960-9822(02)01077-1

60. Xiang X, Saha AK, Wen R, Ruderman NB, Luo Z. AMP-activated protein kinase activators can inhibit the growth of prostate cancer cells by multiple mechanisms. Biochem Biophys Res Commun. (2004) 321:161-7. doi: 10.1016/j.bbrc.2004.06.133

61. Egan D, Kim J, Shaw RJ, Guan K-L. The autophagy initiating kinase ULK1 is regulated via opposing phosphorylation by AMPK and mTOR. Autophagy (2011) 7:643-4. doi: 10.4161/auto.7.6.15123

62. Inoki K, Li Y, Zhu T, Wu J, Guan K-L. TSC2 is phosphorylated and inhibited by Akt and suppresses mTOR signalling. Nat Cell Biol. (2002) 4:648-57. doi: $10.1038 /$ ncb839

63. Meley D, Bauvy C, Houben-Weerts JH, Dubbelhuis PF, Helmond MT, Codogno P, et al. AMP-activated protein kinase and the regulation of autophagic proteolysis. J Biol Chem. (2006) 281:34870-9. doi: 10.1074/jbc.M605488200

64. Karantza-Wadsworth V, Patel S, Kravchuk O, Chen G, Mathew R, Jin S, et al. Autophagy mitigates metabolic stress and genome damage in mammary tumorigenesis. Genes Dev. (2007) 21:1621-35. doi: 10.1101/gad.1565707

65. Dagon Y, Avraham Y, Berry EM. AMPK activation regulates apoptosis, adipogenesis, and lipolysis by eIF2alpha in adipocytes. Biochem Biophys Res Commun. (2006) 340:43-7. doi: 10.1016/j.bbrc.2005.11.159

66. Okoshi R, Ozaki T, Yamamoto $\mathrm{H}$, Ando $\mathrm{K}$, Koida $\mathrm{N}$, Ono S, et al. Activation of AMP-activated protein kinase induces p53-dependent apoptotic cell death in response to energetic stress. J Biol Chem. (2008) 283:3979-87. doi: 10.1074/jbc.M705232200

67. Wang LW, Li ZS, Zou DW, Jin ZD, Gao J, Xu GM. Metformin induces apoptosis of pancreatic cancer cells. World J Gastroenterol. (2008) 14:7192-8. doi: $10.3748 /$ wjg. 14.7192

68. Liang J, Shao SH, Xu ZX, Hennessy B, Ding Z, Larrea M, et al. The energy sensing LKB1-AMPK pathway regulates p27(kip1) phosphorylation mediating the decision to enter autophagy or apoptosis. Nat Cell Biol. (2007) 9:218-24. doi: $10.1038 /$ ncb 1537

69. Blandino G, Valerio M, Cioce M, Mori F, Casadei L, Pulito C, et al. Metformin elicits anticancer effects through the sequential modulation of DICER and c-MYC. Nat Commun. (2012) 3:865. doi: 10.1038/ncomms1859

70. Vazquez-Martin A, Oliveras-Ferraros C, Cufí S, Martin-Castillo B, and Menendez JA. Metformin activates an ataxia telangiectasia mutated (ATM)/Chk2-regulated DNA damage-like response. Cell Cycle (2011) 10:1499-501. doi: 10.4161/cc.10.9.15423

71. Tomic T, Botton T, Cerezo M, Robert G, Luciano F, Puissant A, et al. Metformin inhibits melanoma development through autophagy and apoptosis mechanisms. Cell Death Dis. (2011) 2:e199. doi: 10.1038 /cddis.2011.86

72. Petti C, Vegetti C, Molla A, Bersani I, Cleris L, Mustard KJ, et al. AMPK activators inhibit the proliferation of human melanomas bearing the activated MAPK pathway. Melanoma Res. (2012) 22:341-50. doi: 10.1097/CMR.0b013e3283544929

73. Yuan P, Ito K, Perez-Lorenzo R, Del Guzzo C, Lee JH, Shen $\mathrm{CH}$, et al. Phenformin enhances the therapeutic benefit of BRAF(V600E) inhibition in melanoma. Proc Natl Acad Sci USA. (2013) 110:18226-31. doi: $10.1073 /$ pnas. 1317577110

74. Cerezo M, Tomic T, Ballotti R, Rocchi S. Is it time to test biguanide metformin in the treatment of melanoma? Pigment Cell Melanoma Res. (2015) 28:8-20. doi: $10.1111 /$ pcmr.12267

75. Janjetovic K, Harhaji-Trajkovic L, Misirkic-Marjanovic M, Vucicevic $\mathrm{L}$, Stevanovic $\mathrm{D}$, Zogovic $\mathrm{N}$, et al. In vitro and in vivo antimelanoma action of metformin. Eur J Pharmacol. (2011) 668:373-82. doi: 10.1016/j.ejphar.2011.07.004

76. Niehr F, Von Euw E, Attar N, Guo D, Matsunaga D, Sazegar H, et al. Combination therapy with vemurafenib (PLX4032/RG7204) and metformin in melanoma cell lines with distinct driver mutations. J Transl Med. (2011) 9:76. doi: 10.1186/1479-5876-9-76
77. Pereira FV, Melo ACL, Low JS, De Castro ÍA, Braga TT, Almeida DC, et al. Metformin exerts antitumor activity via induction of multiple death pathways in tumor cells and activation of a protective immune response. Oncotarget (2018) 9:25808-25. doi: 10.18632/oncotarget.25380

78. Cerezo M, Tichet M, Abbe P, Ohanna M, Lehraiki A, Rouaud F, et al. Metformin blocks melanoma invasion and metastasis development in AMPK/p53-dependent manner. Mol Cancer Ther. (2013) 12:1605-15. doi: 10.1158/1535-7163.MCT-12-1226-T

79. Wu CL, Qiang L, Han W, Ming M, Viollet B, He YY. Role of AMPK in UVBinduced DNA damage repair and growth control. Oncogene (2013) 32:2682-9. doi: 10.1038/onc.2012.279

80. Haq R. Metabolic dysregulation in melanoma: cause or consequence? Cancer Discov. (2014) 4:390-1. doi: 10.1158/2159-8290.CD-14-0173

81. Scott DA, Richardson AD, Filipp FV, Knutzen CA, Chiang GG, Ronai $\mathrm{ZA}$, et al. Comparative metabolic flux profiling of melanoma cell lines: beyond the Warburg effect. J Biol Chem. (2011) 286:42626-34. doi: 10.1074/jbc.M111.282046

82. Baenke F, Chaneton B, Smith M, Van Den Broek N, Hogan K, Tang $\mathrm{H}$, et al. Resistance to BRAF inhibitors induces glutamine dependency in melanoma cells. Mol Oncol. (2016) 10:73-84. doi: 10.1016/j.molonc.2015. 08.003

83. Janjetovic K, Vucicevic L, Misirkic M, Vilimanovich U, Tovilovic G, Zogovic $\mathrm{N}$, et al. Metformin reduces cisplatin-mediated apoptotic death of cancer cells through AMPK-independent activation of Akt. Eur. J. Pharmacol. (2011) 651:41-50. doi: 10.1016/j.ejphar.2010.11.005

84. Hirsch HA, Iliopoulos D, Struhl K. Metformin inhibits the inflammatory response associated with cellular transformation and cancer stem cell growth. Proc Natl Acad Sci USA. (2013) 110:972-7. doi: 10.1073/pnas.1221055110

85. Kim SH, Li M, Trousil S, Zhang Y, Pasca Di Magliano M, Swanson KD, et al. Phenformin inhibits myeloid-derived suppressor cells and enhances the anti-tumor activity of PD-1 blockade in melanoma. J Invest Dermatol. (2017) 137:1740-8. doi: 10.1016/j.jid.2017.03.033

86. Scharping NE, Menk AV, Whetstone RD, Zeng X, Delgoffe GM Efficacy of PD-1 blockade is potentiated by metformin-induced reduction of tumor hypoxia. Cancer Immunol Res. (2017) 5:9-16. doi: 10.1158/2326-6066.CIR-16-0103

87. Zabierowski SE, Herlyn M. Learning the ABCs of melanoma-initiating cells. Cancer Cell (2008) 13:185-7. doi: 10.1016/j.ccr.2008.02.015

88. Lehraiki A, Cerezo M, Rouaud F, Abbe P, Allegra M, Kluza J, et al. Increased CD271 expression by the NF-kB pathway promotes melanoma cell survival and drives acquired resistance to BRAF inhibitor vemurafenib. Cell Discov. (2015) 1:15030. doi: 10.1038/celldisc.2015.30

89. Hoek KS, Goding CR. Cancer stem cells versus phenotype-switching in melanoma. Pigment Cell Melanoma Res. (2010) 23:746-59. doi: 10.1111/j.1755-148X.2010.00757.x

90. Ohanna M, Giuliano S, Bonet C, Imbert V, Hofman V, Zangari J, et al. Senescent cells develop a PARP-1 and nuclear factor-\{kappa $\}$ B-associated secretome (PNAS). Genes Dev. (2011) 25:1245-61. doi: 10.1101/gad.625811

91. Leidgens V, Proske J, Rauer L, Moeckel S, Renner K, Bogdahn U, et al. Stattic and metformin inhibit brain tumor initiating cells by reducing STAT3-phosphorylation. Oncotarget (2017) 8:8250-63. doi: 10.18632/oncotarget.14159

92. Montaudié H, Cerezo M, Bahadoran P, Roger C, Passeron T, Machet L, et al. Metformin monotherapy in melanoma: a pilot, open-label, prospective, and multicentric study indicates no benefit. Pigment Cell Melanoma Res. (2017) 30:378-80. doi: $10.1111 / \mathrm{pcmr} .12576$

Conflict of Interest Statement: The authors declare that the research was conducted in the absence of any commercial or financial relationships that could be construed as a potential conflict of interest.

Copyright (c) 2018 Jaune and Rocchi. This is an open-access article distributed under the terms of the Creative Commons Attribution License (CC BY). The use, distribution or reproduction in other forums is permitted, provided the original author(s) and the copyright owner(s) are credited and that the original publication in this journal is cited, in accordance with accepted academic practice. No use, distribution or reproduction is permitted which does not comply with these terms. 